\title{
The metabolic syndrome: progress towards one definition for an epidemic of our time
}

\author{
Paul Zimmet and George Alberti
}

The metabolic syndrome is a major and escalating public-health challenge worldwide. This condition is certainly not benign, since it is associated with a substantially elevated risk not only of type 2 diabetes (fivefold) but also of cardiovascular disease ([CVD] twofold to threefold). The increasing prevalence of metabolic syndrome could reverse the gains made through declining CVD mortality.

Claims that this syndrome was invented by the pharmaceutical industry to boost profits are baseless; the metabolic syndrome has been around for decades. Kylin first described an association between hypertension, hyperglycemia and gout in 1923. In a classic 1947 paper, Vague reported upper-body (android or male-type) adiposity to be the obesity phenotype most commonly associated with metabolic abnormalities linked to raised risks of type 2 diabetes and CVD.

Remarkable interest has been expressed in the metabolic syndrome over the past two decades, in particular because of its increasing frequency against the background of a dramatic rise in obesity and type 2 diabetes mellitus (known as the diabesity epidemic). Nevertheless, confusion and debate have been considerable, owing to a plethora of diagnostic criteria, dissenting views on pathogenesis, and arguments about the clinical usefulness and predictive power of the syndrome. Resolution of this issue seemed to be hampered further by a joint statement from the American Diabetes Association and European Association for the Study of Diabetes, in 2005, which questioned whether the metabolic syndrome really existed, despite the recognized clustering of certain CVD risk factors.

All this debate, however, really amounts to an academic issue; the American Diabetes Association and European Association for the Study of Diabetes experts seem to have missed
The main issue for delbate is not .... whether the metabolic syndrome exists but how to reduce the confusion

P Zimmet is Professor and Director of the International Diabetes Institute, Caulfield South, Melbourne, Australia. G Alberti is Senior Research Fellow, Department of Endocrinology and Metabolism, St Marys Hospital, London, UK.

Competing interests The authors declared no competing interests.

www.nature.com/clinicalpractice doi:10.1038/ncpendmet0834 the point of the syndrome's utility. The main proponents of a definition for the syndrome, the International Diabetes Federation (IDF) and the Adult Treatment Panel, have never claimed that the metabolic syndrome is a good indicator of absolute risk of either diabetes or CVD. Risk engines, such as that developed from the Framingham Heart Study, provide global risk algorithms for this purpose. The IDF does, however, believe that the metabolic syndrome is a good indicator of people at notably raised, long-term risk of developing type 2 diabetes and CVD, for whom lifestyle and other prevention measures might be most beneficial.

The main issue for debate is not, therefore, whether the metabolic syndrome exists but how to reduce the confusion. The two main sources of confusion are whether increased abdominal circumference should be an obligatory component of the syndrome or one of the five optional components, and what waistcircumference measurements should be used. Although the question of abdominal obesity does need to be addressed, we believe that efforts should focus on determining what waist-circumference measurements are relevant to different ethnic groups. Populationspecific waist circumference cut-off points are an essential aspect of the debate, since research has shown that the levels of obesity at which the risk of other morbidities begins to rise varies between populations, most notably in Chinese and South Asian populations.

With these considerations in mind, the Adult Treatment Panel and the IDF have met, under the aegis of the IDF Task Force on Epidemiology and Prevention, to see whether agreement can be reached on one definition and unified criteria for the metabolic syndrome. We welcome this initiative and hope it will highlight what further research is needed. 